\title{
Entscheidungen: Probleme oder Chancen?
}

\author{
Wie Sie proaktiv unangenehme Entscheidungssituationen vermeiden \\ können
}

Entscheidungssituationen werden häufig als Probleme wahrgenommen, die es zu lösen gilt. Allerdings sind Entscheidungen die einzige Möglichkeit, Einfluss auf das zu nehmen, was wichtig ist. Dieser Beitrag grenzt Entscheidungsprobleme und Entscheidungschancen ab und zeigt auf, wie durch proaktives Entscheiden nicht nur die Wahrscheinlichkeit, mit Problemen konfrontiert zu werden, reduziert werden kann, sondern auch systematisch attraktive Entscheidungschancen identifiziert werden können.

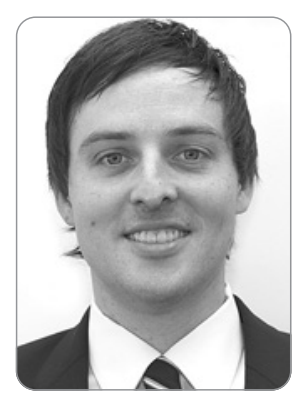

Prof. PD Dr. habil. Johannes Ulrich Siebert ist Professor am Management Center Innsbruck und Privatdozent an der Universität Bayreuth. Bevorzugte Forschungsgebiete: Behavioral OR, Entscheidungstheorie, Value-focused Thinking, Behavioral 0M/ SCM.

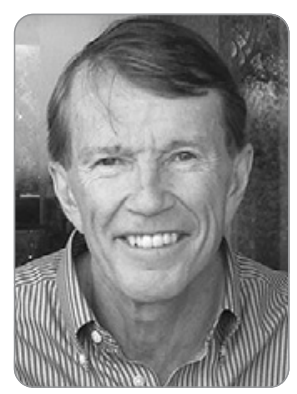

\section{Prof. Dr. Ralph L. Keeney}

ist Research Professor Emeritus an der Duke University (USA). Bevorzugte Forschungsgebiete: Decision Sciences, Value-focused thinking.
Summary: Many individuals and organizations face and address decision situations as problems, which are to be solved. However, decisions are the only way to influence actively what is important. This paper differentiates decision problems and decision opportunities and illustrates how proactive, value-focused decision making can create attractive decision opportunities and help reduce the chance of some unwanted decision problems.

Stichwörter: Entscheidungstheorie, proaktives Entscheiden, Value-focused Thinking, Entscheidungsproblem, Entscheidungschance
1. Entscheidungssituationen werden als Probleme wahrgenommen

Entscheidungen sind die einzige Möglichkeit, Einfluss auf das zu nehmen, was für Sie oder Ihre Organisation wichtig ist (Keeney, 2020). Dennoch werden Entscheidungssituationen häufig als Probleme wahrgenommen und angegangen. Sowohl in der Forschung als auch in der Praxis hat sich dieses auch sprachlich verankert - es wird von "Entscheidungsproblemen" gesprochen. Gründe dafür gibt es viele. Beispielsweise bedeutet das Treffen einer Entscheidung auch das Übernehmen von Verantwortung, die Einschränkung zukünftiger Entscheidungsfreiheit (wer sich heute ein Haus kauft, setzt dafür finanzielle Mittel ein, die morgen nicht für den Kauf eines Autos eingesetzt werden können) oder schlicht den kognitiven Aufwand, die Entscheidung sauber zu durchdenken. In der Folge tendieren viele Menschen und Organisationen dazu, Entscheidungen vor sich herzuschieben („Prokrastination“).

Viele Entscheidende, sowohl auf individueller als auch auf organisationaler Ebene, begnügen sich mit dem Status Quo. Und das selbst dann, wenn sie nicht wirklich zufrieden sind oder sogar wahrnehmen, dass sich dieser sukzessive verschlechtert. Es wird reagiert und nicht proaktiv agiert. Ehepaare gehen beispielsweise im Durchschnitt sieben Jahre zu spät zu einer Eheberatung. Dann aber ist der Leidensdruck bereits sehr hoch und es liegt zumeist ein "schwer zu lösendes Problem" vor.

Das nachfolgende Beispiel skizziert eine Situation, die heutzutage viele Familien treffen kann, wenn sie (über einen längeren Zeitraum) reaktiv in ihren Entscheidungssituationen agieren: 
Maria und Anton sind beide 55 Jahre alt und leben in einer kleinen Stadt in Tirol. Sie haben und sorgen für drei Kinder, von denen eines noch zur Schule geht und zwei studieren. Vor 25 Jahren hat sich das Paar dazu entschlossen, dass Maria, gelernte Bürokauffrau, ihren Job aufgibt und sich um den Haushalt und die Erziehung der Kinder kümmert, während Anton, gelernter Industriemechaniker, als Alleinverdiener fungiert. Anton ist angestellt bei einem mittelständischen Automobilzulieferer mit 800 Mitarbeitern, von denen 200 als Industriemechaniker*innen arbeiten.

Ein internationales Großunternehmen kauft das Unternehmen in Tirol auf und kündigt kurzfristig an, die Produktion nach Asien zu verlagern. In der Folge verliert Anton seinen Job und die Familie ihre Existenzgrundlage. Anton hat sehr große Schwierigkeiten einen neuen Job zu bekommen, insbesondere da nicht nur er, sondern auch 199 andere Industriemechaniker*innen mit ähnlichen Qualifikationen - und viele von ihnen jünger und damit billiger - einen neuen Job suchen. Ohne das Einkommen von Anton können die Eltern die Ausbildung der Kinder nicht unterstützen. Die Familie kann sich das große auf Kredit gekaufte Haus nicht mehr leisten und muss in der Folge umziehen. Anton fühlt sich als Versager, weil er nicht mehr für die Familie sorgen kann und sitzt griesgrämig zu Hause auf dem Sofa. Dies belastet die Beziehung zu seiner Frau erheblich. In einer solchen Situation sind schon viele Familien regelrecht zerbrochen.

Es ist offensichtlich, dass die Familie ein schwerwiegendes

Problem hat. Viele Menschen hadern in einer solchen Situation mit dem Schicksal und schlussfolgern, dass sie einfach Pech gehabt haben, da sie ja nichts dafürkönnen, wenn beispielsweise ihr Unternehmen verkauft und die Produktion verlagert wird sowie Mitarbeiter*innen entlassen werden. Es stimmt, dass üblicherweise die Mitarbeite$\mathrm{r}$ *innen kaum Einfluss darauf haben, ob ihr Unternehmen verkauft wird. Auf individueller Ebene bestehen jedoch sehr wohl Möglichkeiten aktiv Einfluss auf das eigene Leben zu nehmen, beispielsweise die Wahrscheinlichkeit hinzufallen (i.e. entlassen zu werden) signifikant zu reduzieren und, wenn das schon nicht vermieden werden kann, zumindest die Wahrscheinlichkeit, schnell wieder auf die eigenen Füße zu kommen (i.e. schnell einen angemessenen neuen Job zu finden) signifikant zu erhöhen.

Dieser Beitrag grenzt Entscheidungsprobleme und Entscheidungschancen ab und zeigt auf, wie durch proaktives Entscheiden nicht nur die Wahrscheinlichkeit, mit Problemen konfrontiert zu werden, reduziert werden kann, sondern auch, wie systematisch attraktive Entscheidungschancen identifiziert werden können.

\section{Value-focused Thinking vs. Alternative-focused Thinking}

Der typische und in der Praxis dominierende Entscheidungsprozess lässt sich in vier Schritte unterteilen. Im ersten Schritt wird das Entscheidungsproblem identifiziert. Häufig ist dabei die Unzufriedenheit mit der aktuellen Situation verbunden mit dem Wunsch nach Verbesserung der Auslöser. Im zweiten Schritt suchen Entscheidende nach Lösungen und entwickeln zügig, mit wenig Aufwand einige zumeist "offensichtliche“ Alternativen. Als Ausgangspunkt dienen dabei zumeist eine oder mehrere bekannte Alternative(n). Wenn es beispielsweise einer Familie im letzten Jahr in Griechenland auf Korfu gefallen hat, werden auch bei der nächsten Urlaubsplanung bei gleichen Interessenlagen und Rahmenbedingungen ähnliche Alternativen entwickelt, beispielsweise Korfu, Rhodos, Samos oder Kreta. Die Nordsee, Spanien und die Türkei werden jedoch nicht als Alternativen identifiziert. Es kann auch passieren, dass sich eine unvorhersehbare Alternative auftut und eine Entscheidung zwischen dieser und dem Status Quo abverlangt. In beiden Fällen wählen Entscheidende im dritten Schritt einige Kriterien aus, die die aus ihrer Sicht relevanten Folgen der Alternativen abbilden. Im vierten Schritt überprüfen Entscheidende die gesammelten Informationen und treffen auf deren Basis ihre Entscheidung.

Keeney (1992) hat für die sehr enge Orientierung an offensichtlichen Alternativen im Entscheidungsprozess den Begriff Alternative-focused Thinking (deutsch = Alternativen-fokussiertes Denken) geprägt. Alternative-focused Thinking ist für viele die „natürliche“ Art und Weise, Entscheidungen zu treffen. Bereits die ersten Entscheidungen in frühester Kindheit, wie beispielsweise die Entscheidung zwischen Eis oder Schokolade, sowie die Erfahrungen mit Entscheidungen haben viele Menschen dahingehend konditioniert, Entscheidungen vordergründig als Wahl zwischen gegebenen Alternativen zu betrachten. Trotz der weiten Verbreitung weist das Alternative-focused Thinking bedeutende Unzulänglichkeiten auf. Die Auswahl aus offensichtlichen Alternativen scheint zwar das Entscheidungsproblem auf den ersten Blick mit relativ wenig Aufwand $\mathrm{zu}$ "lösen“. Bei genauerer Betrachtung ist jedoch festzustellen, dass das Ziel nicht darin bestehen kann, reaktiv einfach nur Entscheidungsprobleme zu lösen, sondern mit Hilfe von Entscheidungen proaktiv Einfluss auf die Umwelt zu nehmen. In diesem Zusammenhang ist es eher als Zufall zu bezeichnen, wenn sich unter den offensichtlichen Alternativen die bestmögliche befände (Siebert und Keeney, 2015). Ursächlich dafür ist, dass die Identifikation von Alternativen im "luftleeren Raum", ohne Verbindung zu den Werten des Entscheidungsträgers, geschieht (Keeney, 1992, S. 44 ff.). 
Genau an diesem Punkt setzt Value-focused Thinking an ("Values" haben im Englischen eine weiter gefasste Bedeutung als "Werte“ im Deutschen, die insbesondere auf moralische und ethische Aspekte abstellen. Daher findet der englische Begriff Anwendung). Values bezeichnen das, was einem Menschen wichtig ist, und sind fundamental für alles, was ein Mensch unternimmt. Daher sollten Values die treibende Kraft bei einer Entscheidung sein. Die Values der Menschen sind der Grund, warum für Entscheidungen Zeit und Verstand eingesetzt werden sollten (vgl. Keeney, 1996, S. 537). Value-focused Thinking impliziert im Vergleich zum Alternative-focused Thinking einen Paradigmenwechsel, der sich in drei Aspekten äußert. Erstens wird ein signifikanter Aufwand für das Bewusstwerden, Explizieren und Verdeutlichen der Values betrieben. Dabei dienen logische Konzepte und Methoden zur Identifikation und Strukturierung von Zielen. Zweitens erfolgt die Artikulation der Werte und Ziele zu Beginn des Entscheidungsprozesses. Drittens dienen die artikulierten Ziele explizit der Entwicklung von Alternativen und der Identifikation von Entscheidungschancen (vgl. Keeney, 1996, S. 537 f.).

In den letzten Jahren wurden zahlreiche empirische Studien durchgeführt, die für die Notwendigkeit und die Wirksamkeit von proaktivem Agieren in Entscheidungssituationen sprechen. Bond et al. (2008) zeigten, dass sich viele Entscheidende gerade einmal der Hälfte ihrer Ziele in einer Entscheidungssituation bewusst sind. Doch wie soll eine gute Entscheidung getroffen werden, wenn einem die $\mathrm{zu}$ erreichenden Ziele nicht einmal bewusst sind? Siebert und Keeney (2015) haben in einer Studie die Fähigkeit zur Entwicklung von Alternativen untersucht. Die Teilnehmenden waren Studierende. Die Entscheidungssituation war, welche Alternativen dazu beitragen können, die Ziele während eines Praktikums zu erreichen, wie beispielsweise später einen Job angeboten zu bekommen, sich bestimmte Fähigkeiten anzueignen oder sich zu vernetzen. Die Probanden befanden sich in einer Phase ihres Studiums, in der sie schon ein Praktikum absolviert hatten oder gerade ein Praktikum planten. Daher ist davon auszugehen, dass sie sich bereits intensiv mit der Entscheidungssituation auseinandergesetzt hatten. Selbst diese Teilnehmenden konnten nur ca. ein Drittel der für sie relevanten Alternativen eigenständig identifizieren. Über die Hälfte der Teilnehmenden übersah zunächst sogar die für sie bestmögliche Alternative. In vier weiteren Studien konnten die Autoren zeigen, dass der Einsatz von Zielen als Stimulus zu mehr und besseren Alternativen führt. Bei der Aufgabe, einen Titel für eine wissenschaftliche Arbeit zu finden, entwickeln erfahrenere Teilnehmende wie Masterstudierende und Doktorandinnen und Doktoranden normalerweise bessere Alternativen als Bachelorstudierende. Aber mit Zielen stimulierte Bachelorstudierende konnten ähnlich gute Alternativen wie im wissenschaftlichen Arbeiten erfahrenere, aber nicht mit Zielen stimulierte Masterstudierende und Doktorandinnen und Doktoranden identifizieren (Siebert, 2016).

Proaktives, „value-focused“ Entscheiden von Individuen kann hinsichtlich ihres Grads an Proaktivität in Entscheidungssituationen in sechs Dimensionen beschrieben werden. Die Dimensionen "Initiative zeigen" und "nach Verbesserungen streben" beschreiben die Aspekte proaktiver Persönlichkeitseigenschaften. Die Dimensionen "systematische Identifikation von Zielen“, „zielgerichtete Erhebung von Informationen“, „zielgerichtete Entwicklung von Alternativen“ und der "Einsatz eines Entscheidungsradars“ messen die proaktiven kognitiven Fähigkeiten von Individuen (Siebert und Kunz, 2016). Mit proaktivem Entscheiden lassen sich nicht nur ca. $50 \%$ der Streuung der Entscheidungszufriedenheit, sondern auch ca. $35 \%$ der Streuung der Lebenszufriedenheit erklären (Siebert et al., 2020). Wer also proaktiver in seinen Entscheidungen agiert, ist im Durchschnitt zufriedener mit seinem oder ihrem Leben. Wenn Sie die in diesem Beitrag diskutierten Unterschiede zwischen Entscheidungsproblemen und Entscheidungschancen verinnerlichen und verstärkt Entscheidungschancen suchen, dann agieren Sie proaktiver in Ihren Entscheidungssituationen. Damit erhöhen Sie Ihre Chance signifikant, langfristig zufriedener mit Ihrem Leben sein!

\section{Entscheidungsprobleme vs. Entscheidungschancen}

Die Unterscheidung zwischen Entscheidungsproblemen und Entscheidungschancen ist wichtig, aber ist den meisten Entscheidenden nicht bewusst.

Entscheidungsprobleme sind Entscheidungen, die jemand als Folge von Entscheidungen anderer oder nicht beeinflussbarer äußerer Umstände, die nicht selbst kontrolliert werden, treffen muss. („Decision problems are decisions that you must face as a result of others' decisions and/or circumstances beyond your control." Keeney, 2020). Üblicherweise werden diese Probleme so adressiert, dass sie "gelöst" werden. Häufig begnügen sich die Entscheidenden dabei mit den offensichtlichen, in der Vergangenheit in ähnlichen Situationen gewählten oder von anderen vorgeschlagenen Alternativen.

Wenn jemand den Job aufgrund einer Reorganisation verliert, entsteht sicherlich ein Problem und erfordert eine Entscheidung oder mehrere Entscheidungen, um einen neuen Job zu finden. Was wäre jedoch, wenn ein unerwartetes Angebot für einen Job in einem interessanten Unternehmen, der einen echten Karrieresprung bedeutet, an einen herangetragen wird? Auch in dieser Situation müssen Entscheidungen getroffen werden. Aber stellen die ein Problem dar? Es ist keine negative, sondern eine ausgesprochen positive Situation. Aber es ist immer noch ein 
Entscheidungsproblem, weil auf eine Entscheidung einer anderen Person oder Organisation reagiert werden muss (Keeney, 2020).

Entscheidungschancen sind Entscheidungssituationen, die bewusst von den Entscheidenden kreiert werden und über die die Entscheidenden die Kontrolle haben. Um Entscheidungschancen $z u$ verstehen, ist folgende Frage hilfreich: Wer soll Ihre Entscheidungen treffen? Die Antwort dabei ist trivial, denn Sie sollten Ihre Entscheidungen treffen. Die Antwort auf die nächste Frage ist es für viele hingegen nicht: Wer soll entscheiden, mit welchen Entscheidungen Sie sich auseinandersetzen? Idealerweise sind auch das Sie; vielleicht nicht immer, aber möglichst oft. Denn nur dadurch können Sie aktiv Einfluss auf alles nehmen, was Ihnen wichtig ist (Keeney, 2020).

Als Beispiel, welches für viele Menschen Relevanz hat, dient im Folgenden der Berufspendler Paul, der jeden Tag mehr als eine Stunde Zug fährt. Er hat sich angewöhnt, währenddessen mit dem Handy zu spielen oder im Internet zu surfen, also letztlich die "Zeit totzuschlagen“. Was kann er tun um, diese Zeit besser zu nutzen? Er könnte etwas machen, was ihn wirklich interessiert, entspannt, ihm Zeit spart, Spaß macht, etc. Für Paul liegt vorher kein Problem vor. Aber er kann eine Entscheidungschance proaktiv kreieren, indem er sich fragt, wie er die Zeit besser nutzen könnte.

\section{Reaktives vs. proaktives Agieren in Entscheidungssituationen}

Die Unterschiede zwischen reaktivem, alternative-focused und proaktivem, value-focused Entscheiden lässt sich hervorragend am Beispiel der Gesundheit von Claudia darstellen. Zunächst ist bei der reaktiv entscheidenden Claudia alles in Ordnung, dann knickt sie beim Fußball spielen um und verletzt sich ihr Außenband am Fuß. Sie hat ein Entscheidungsproblem: Zu welcher Ärztin möchte sie gehen? Anschließend hat die Ärztin ein Entscheidungsproblem: Welche Tests sollen zur Diagnose eingesetzt werden? Sodann hat Claudia mit Unterstützung der Ärztin das Entscheidungsproblem: Welche Behandlung soll angewandt werden? Wenn alles gut geht, kann Claudia nach ein paar Wochen wieder Fußball spielen. In ein paar Monaten knickt sie wieder um und das Prozedere wiederholt sich, so wie jede Saison. Sie hat einfach immer nur Pech, oder? Nein, nicht zwangsläufig. Sie hätte sich einmal proaktiv mit ihren Fußverletzungen auseinandersetzen können, als sie gerade einmal fit war. Ihre Ziele sind offensichtlich: sie möchte weiterhin Fußball spielen, aber Fußverletzungen vermeiden. Welche Möglichkeiten gibt es, dass sie weniger oft umknickt? Zahlreiche! Beispielsweise gibt es Spezialschuhe oder orthopädische Bandagen. Darüber hinaus könnte sie auch Übungen machen, die ihre Muskeln und Sehnen stärken oder nicht mehr auf Kunstrasen spielen, wenn sie immer dort und nicht auf Naturrasen umknickt.

Viele Studien bringen den Körperfettgehalt, wenig Bewegung, hohen Alkoholkonsum oder Rauchen langfristig in Verbindung mit teilweise erheblichen gesundheitlichen Problemen. Reaktiv Entscheidende warten ab, bis eines dieser möglichen Probleme akut wird. Dann sehen sie sich mit einem drastischen Problem konfrontiert, das es zu lösen gilt, beispielsweise welche Herz-OP die sinnvollste ist. Proaktiv Entscheidende antizipieren hingegen mögliche Probleme in der Zukunft, betreiben Prävention - beispielsweise durch ein bisschen mehr Bewegung, weniger Alkohol, gesündere Enährung, etc. Wichtig ist darauf hinzuweisen, dass es keinen Automatismus gibt. Man kann tatsächlich "Pech haben“. Durch proaktives Agieren in Entscheidungssituation kann aber die Wahrscheinlichkeit reduziert werden, dass ein ungewünschter Zustand eintritt.

\section{Einfluss von Entscheidungssituation auf die Lebensqualität}

Ein wesentlicher Unterschied zwischen Entscheidungsproblemen und Entscheidungschancen liegt in ihrer Wirkung auf das, was wichtig ist - im Falle von Individuen und im Folgenden näher betrachtet: die Lebensqualität (als allgemeines Konstrukt; je nach Fall eignen sich spezifischere Konstrukte wie Zufriedenheit, Fitness, Gesundheit, Einkommen, etc.).

Bei Entscheidungsproblemen wird die Lebensqualität durch etwas, das außerhalb der Kontrolle des Entscheidenden liegt, reduziert, beispielsweise das Auto ist kaputt, der Urlaubsantrag wird abgelehnt, ein Orkan deckt das Dach des Eigenheims ab, man bekommt eine Grippe, etc. Die Lebensqualität reduziert sich. Wenn der Leidensdruck groß genug ist, widmen sich die Entscheidenden dem Entscheidungsproblem und versuchen es zu lösen: das Auto wird repariert, das Haus gedeckt, man geht zum Arzt, etc. In Abb. 1 sind die durchschnittliche Lebensqualität sowie deren Bandbreite über vier Meilensteine von Entscheidungssituationen (Entscheidungssituation erkennen, Alternative wählen, Alternative implementieren, vom Entscheidungsergebnis beeinflusst werden) für Entscheidungsprobleme auf der linken Seite exemplarisch dargestellt. Wenn das Problem gelöst wurde, wird üblicherweise die Lebensqualität mehr oder weniger wiederhergestellt. Im Durchschnitt wird sogar ein geringeres Level angenommen (Keeney, 2020). Wenn beispielsweise Claudias Bänder im Fuß regelmäßig reißen, dann ist wohl nicht davonauszugehen, dass sich die Funktionsweise des Fußes insgesamt verbessert.

Bei Entscheidungschancen bleibt die Lebensqualität konstant, wenn nichts unternommen wird. Wenn Claudia aber 


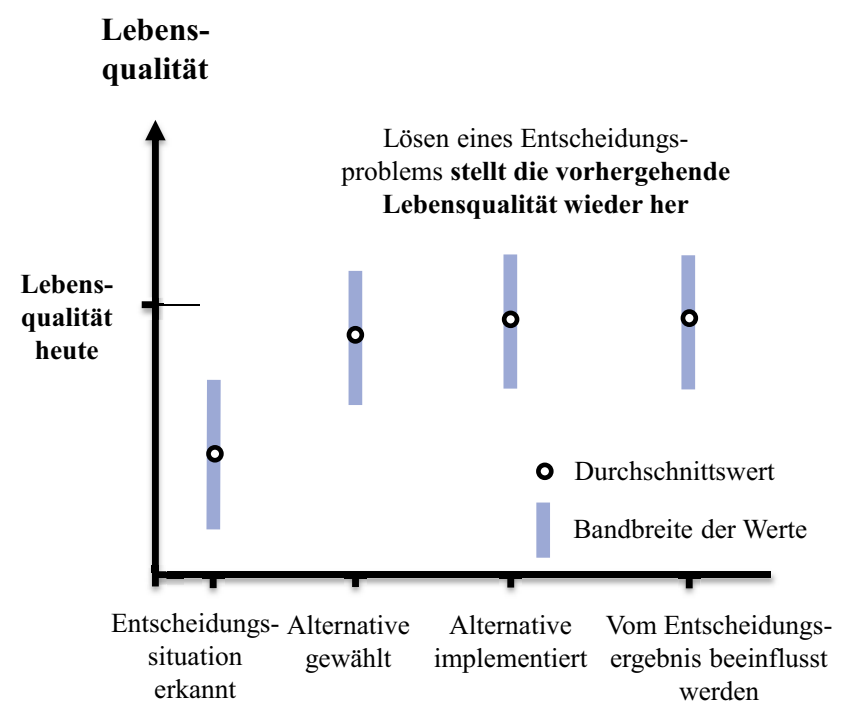

Meilensteine von Entscheidungssituationen

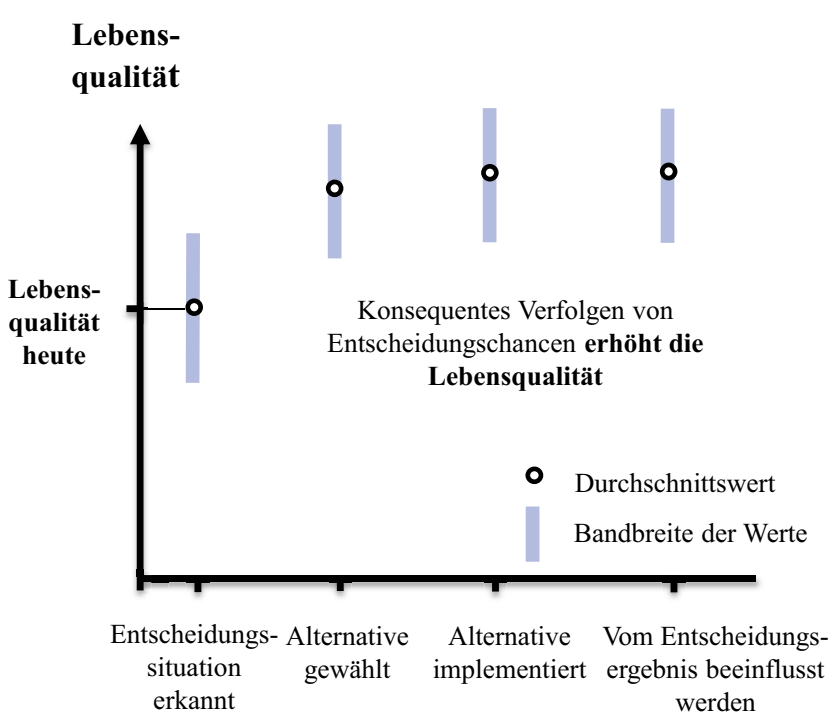

Meilensteine von Entscheidungssituationen

Abb. 1: Die Lebensqualität im Zeitablauf für Entscheidungsprobleme und Entscheidungschancen basierend auf Siebert und Hannes (eingereicht).

beispielsweise beschließt, durch Physiotherapie und regelmäßige Gymnastik ihre Bänder und Muskeln in ihrem Fuß zu stärken, erreicht sie Schritt für Schritt eine bessere Gesundheit und damit höhere Lebensqualität und reduziert die Wahrscheinlichkeit, dass ihre Bänder reißen. In Abb. 1 sind die durchschnittliche Lebensqualität sowie deren Bandbreite für Entscheidungschancen auf der rechten Seite schematisch dargestellt. Es wird angenommen, dass sich die Lebensqualität im Durchschnitt erhöht (Keeney, 2020).

\section{Wie Probleme proaktiv vermieden werden können}

Die Vermeidung bzw. die Minimierung der Eintrittswahrscheinlichkeit von unangenehmen Entscheidungssituationen durch aktive Prävention ist im Kontext von Gesundheit bei vielen Menschen zu sehen. Auch in anderen Bereichen betreiben Menschen Prävention, schließen zahlreiche Versicherungen ab oder treffen andere Vorkehrungen. Und das auch dann, wenn die Eintrittswahrscheinlichkeiten extrem gering sind, wie beispielsweise Überflutung, Diebstahl, Verkehrsunfall oder einen größten anzunehmenden Unfall (GAU) in einem Kernreaktor.

Diese Präventionsmaßnahmen bzw. Alternativen sind offensichtlich und werden größtenteils aktiv an die Entscheidenden herangetragen, beispielsweise durch Versicherungsvertreter*innen oder Verkäufer*innen von Survival-kits oder Atombunkern für den häuslichen Garten. Die Entscheidenden können letztlich mit relativ wenig Aufwand die für sich passende Alternative auswählen. Wenn es keine solchen Alternativen gibt, verharren viele Menschen reaktiv in ihrem Status Quo bis ggf. durch ein Ereignis außerhalb der Kontrolle des Entscheidenden die Lebensqualität stark vermindert wird. Die Folgen eines solchen Verhaltens können dramatisch sein und das Leben nachhaltig verändern.
Im Folgenden wird das Beispiel des reaktiven Alleinverdieners Anton aus Tirol wieder aufgegriffen und gezeigt, wie das Problem hätte gelindert oder gar vermieden werden können. Dafür hätten Anton und Maria bereits von 25 Jahren anfangen müssen, systematisch proaktiv in ihren Entscheidungssituationen zu agieren und zu überlegen, welche Alternativen ihnen helfen, ihre Ziele zu erreichen.

Als Ausgangspunkt ist es dafür zunächst erforderlich, die Ziele von Anton und Maria zu identifizieren. Dazu bietet es sich beispielsweise an, darüber nachzudenken, welche Konsequenzen in ihrem Leben wünschenswert bzw. nicht wünschenswert sind. Sicherlich stellt es für die Familie eine gravierende Konsequenz dar, wenn die finanzielle Existenzgrundlage entfällt. Daraus lässt sich das Fundamentalziel "Finanzielle Sicherheit maximieren" ableiten. Neben diesem Ziel gibt es sicherlich noch weitere relevante Ziele. Um den Rahmen dieses Beitrags nicht zu sprengen, wird die proaktive Vorgehensweise in Antons und Marias Entscheidungssituationen nur anhand dieses einen Ziels illustriert. In der Praxis ist es jedoch empfehlenswert, auch die anderen Ziele schrittweise heranzuziehen. Die Frage lautet daher: Welche Möglichkeiten hätte die Familie gehabt, das Ziel "Finanzielle Sicherheit maximieren“ besser zu erreichen, auch wenn Anton ggf. entlassen wird?

- Maria hätte eine Teilzeitstelle annehmen können, die sie bei Bedarf aufstocken kann

- Maria hätte - zumindest in den letzten 10 Jahren - sich weiterqualifizieren und im Homeoffice arbeiten können

- Die studierenden Kinder könnten ihr Studium zumindest teilweise selbst durch Stipendien oder Nebenjobs finanzieren, die sie bei Bedarf aufstocken können

- Die Familie hätte vielleicht ein kleineres Haus kaufen können, für das sie sich nicht so stark verschuldet hätten 
Im Folgenden sollen die Möglichkeiten von Anton, dem Alleinverdiener, der in der obigen Aufzählung noch nicht berücksichtigt wurde, detaillierter betrachtet werden. Dabei liegt der Fokus auf den beruflichen Aktivitäten: Wie können Arbeitnehmer*innen das Fundamentalziel "Finanzielle Sicherheit maximieren" erreichen? Beispielsweise durch das Verfolgen der Ziele "Wahrscheinlichkeit entlassen $\mathrm{zu}$ werden minimieren" und "Wahrscheinlichkeit schnell einen angemessenen neuen Job zu finden maximieren“. Die Frage lautet daher: Welche Möglichkeiten hätte Anton gehabt, diese Ziele besser zu erreichen?

- Anton hätte sich kontinuierlich fortbilden können. Dabei hätte er überlegen können, welche Fähigkeiten in der $\mathrm{Zu}$ kunft in seinem Berufsfeld besondere Relevanz erlangen könnten und sich genau diese aneignen, beispielsweise IT, Robotik, Teamführung, Englisch, etc.

- Anton hätte sich in seinem Fachbereich vernetzen können, beispielsweise durch Teilnahme an Veranstaltungen mit Arbeitnehmer*innen und Arbeitgeber*innen in seinem Fachbereich

- Anton hätte sich kontinuierlich und damit im konkreten Fall frühzeitig über potenzielle Arbeitgeber*innen informieren können

Diese Aufzählung ist mitnichten vollständig. Diese Möglichkeiten hätte er auch nicht gleichzeitig ergreifen müssen. Vermutlich hätten aber 1-2 Stunden pro Woche mehr als gereicht! Eine solche Investition erscheint im Vergleich $\mathrm{zu}$ den möglichen negativen Konsequenzen sehr sinnvoll. Viele Alleinverdienende wie Anton würden heutzutage sicherlich im Nachhinein gerne einen solchen Aufwand getrieben haben. Darüber hinaus hätten die verbesserten Fähigkeiten auch dazu beitragen können, befördert zu werden, so dass sich die "Investition" allein schon damit amortisiert hätte.

\section{Fazit: Wie Sie bekommen, was Sie wollen}

Viele Individuen und Organisationen sehen in Entscheidungen Probleme, die es zu lösen gilt. Diese Grundhaltung ist reaktiv zu bezeichnen und fokussiert zumeist auf die Wiederherstellung des Zustands, bevor das Problem erkannt wurde. Im Gegensatz schlägt Keeney (1992, 2020) vor, proaktiv in Entscheidungssituationen $\mathrm{zu}$ agieren und systematisch Entscheidungschancen zu identifizieren. Das Beispiel um die Familie von Anton aus Tirol zeigt, wie proaktives, value-focused Entscheiden die Wahrscheinlichkeit für das Eintreten von Entscheidungssituationen mit erheblichen negativen Konsequenzen im Sinne einer Prävention reduzieren kann.

Die gleiche proaktive Denkweise („value-focused Thinking“) kann auch die Wahrscheinlichkeit erhöhen, dass Sie erreichen, wovon Sie träumen. Dafür müssen Sie Ihre
Werte und Ziele identifizieren und diese systematisch dazu einsetzen, Entscheidungschancen zu kreieren. Viele Studierende sehen beispielsweise ihre Abschlussarbeit als eine lästige Pflicht, also ein Problem an. "Reframen“ Sie dieses Entscheidungsproblem als eine Entscheidungschance. Identifizieren Sie Ihre Ziele. Wenn Sie beispielsweise unbedingt bei einer bestimmten Organisation arbeiten wollen, können Sie überlegen, wie Sie Ihre Abschlussarbeit dafür instrumentalisieren können. Vielleicht gibt es Problemstellungen in diesem Unternehmen, die sich für eine Abschlussarbeit eignen. Vielleicht identifizieren Sie in einer solchen Arbeit einen akuten Handlungsbedarf in einem aufkommenden Feld, in dem Sie die entsprechende Expertise mitbringen (u. a. durch Ihre Abschlussarbeit). Oder Sie überlegen, ggf. eine wissenschaftliche Karriere anzustreben. Dann können Sie Ihre Abschlussarbeit so ausgestalten, dass Sie eng mit Ihrem Betreuenden zusammen an einer aktuell wissenschaftlich relevanten Forschungsfrage arbeiten, Sie dadurch das Lehrstuhlteam kennenlernen und erkennen, ob Ihnen so etwas Spaß machen könnte und idealerweise schon die Grundlagen für Ihre erste Publikation legen. Andere Beispiele für Entscheidungschancen Studierender liegen in der Wahl des Studienfachs, der Spezialisierung oder eines Auslandsaufenthalts. Das proaktive Verfolgen von Entscheidungschancen erhöht nicht nur die Lebensqualität, sondern auch die Befähigung („Empowerment“) das eigene Leben aktiv zu gestalten. Take advantage of this opportunity!

\section{Literatur}

Bond, S. D., Carlson, K.A., Keeney, R. L., Generating Objectives: Can Decision Makers Articulate What They Want?, in: Management Science, Vol. 54 (2008), 56-70.

Keeney, R. L., Value-focused Thinking, A Path to Creative Decision Making, Cambridge 1992.

Keeney, R. L., Value-focused thinking: Identifying decision opportunities and creating alternatives, in: European Journal of Operational Research, Vol. 92(3)3 (1996), 537-549.

Keeney, R. L., Give Yourself a Nudge: Practical Procedures for Making Better Personal and Business Decisions, Cambridge 2020.

Siebert, J. U., Can Novices Create Alternatives of the Same Quality as Experts?, in: Decision Analysis, Vol. 13(4) (2016), 278-291.

Siebert, J. U.; Hannes, C., The relative benefits of pursuing decision opportunities and decision problems (eingereicht).

Siebert, J. U., Keeney, R. L., Creating More and Better Alternatives for Decisions Using Objectives, in: Operations Research, Vol. 63 (2015), 11441158.

Siebert, J. U., Kunz, R., Developing and Validating the Multidimensional Proactive Decision-Making Scale. In: Sonderheft "Behavioral Operations Research“, European Joumal of Operational Research, 249(3) 2016, 864877.

Siebert, J. U., Kunz, R., Rolf, P., Effects of proactive decision making on life satisfaction. European Journal of Operational Research, 280(1) (2020), 1171-1187.

Siebert, J. U., von Nitzsch. R., Das Jobauswahlproblem für Berufseinsteiger: Eine entscheidungstheoretische Anwendung - Teil 1: Problemstrukturierung in Ziele, Alternativen und Unsicherheiten, in: Wissenschaftliche Beiträge, Wirtschaftswissenschaftliches Studium, Vol. 47(10) (2018), 4-11. 